\title{
Consistent World Models for Cooperating Robots: Separating Logical Relationships, Sensor Interpretation and Estimation
}

\author{
Andreas Schierl, Andreas Angerer, Alwin Hoffmann and Wolfgang Reif \\ Institute for Software and Systems Engineering, University of Augsburg, Augsburg, Germany \\ \{schierl, angerer, hoffmann, reif\}@isse.de
}

\begin{abstract}
When working with multiple independent mobile robots, each has a different knowledge about its environment, based on its available sensors. This paper proposes an approach that allows working with these different views by independently modeling the common logical relationships between the elements in the scene and the meaning of device-specific sensor data. Using these models, for each robot an estimation process can automatically be derived that combines and processes the available information to fill in the unknown geometric relationships between the elements, while reacting to changes in the logical relationships. The proposed approach facilitates the consistent coordination of the robots through an application that works on a common world model, while for execution each robot uses its available data and estimations. The approach is demonstrated in a case study of two cooperating mobile robots that pick up an object and hand it over while passing each other.
\end{abstract}

\section{INTRODUCTION}

Working with multiple, independent mobile robots, each has a different knowledge about its environment. These knowledge differences are based on the sensors available to the individual robots: While for typical industrial robots the positions of workpieces and tasks are exactly defined and ensured through fixtures, mobile robots often work in an environment where this strict structure is not present. In conjunction with the low precision of mobile robot locomotion, geometric uncertainty exists to an extent so that has to be handled. Therefore, sensors are added - either integrated into the robot, or mounted in the environment to resolve the uncertainty based on measurements, and to consistently update the world model - the representation of the application's beliefs about its environment - accordingly. In popular approaches, the processing of sensor data is explicitly implemented or configured for the given scenario to form the robot's world model.

In contrast, this paper proposes to independently define the logical model (consisting of the logical relationships) and the measurement model (defining the meaning of sensor measurements in a geometric context). Based on these definitions and available sensor data, the proposed framework derives an estimation pipeline for the current situation. This estimation pipeline is created at run-time and provides consistent data for different robots that are controlled from the same application, each working with its available data.
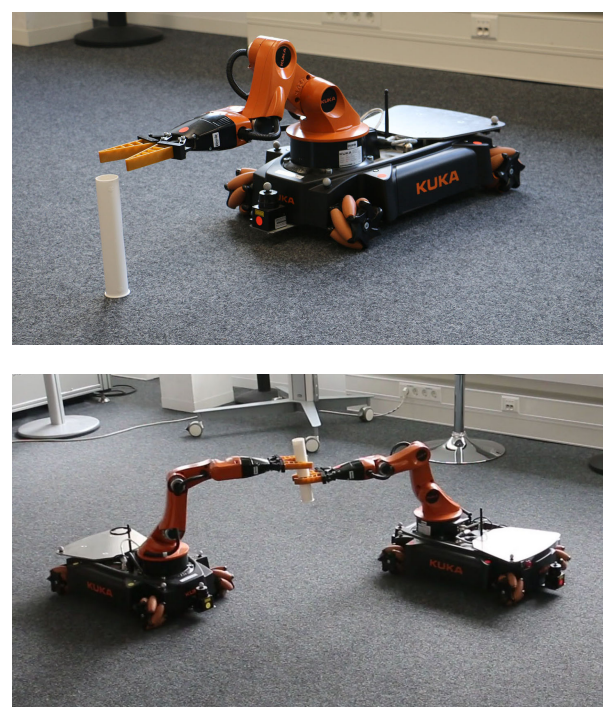

Figure 1. Handing over an object between two moving youBots.

Hence, the main contribution of this paper is a proposed separation of logical relationships and sensor interpretation which allows automatic derivation an estimation pipeline. In doing so, the estimation process no longer has to be configured manually, but emerges from the given relationships and measurements. Additionally, the estimation pipeline can automatically adapt to changes in the environment. For example, when a robot grasps an object, sensor data no longer defines the position of the object in the environment, but rather its position in the gripper.

As a further contribution, this approach allows consistent world models between cooperating mobile robots, where a common logical model is used, while for each robot its available sensors are used to derive information about geometric aspects it knows about. This offers better reusability, because common logical models, but also definitions of sensor interpretations can be used in other contexts, independent from the exact estimations they yield there.

As a real-world example, the interaction of two mobile robots is analyzed. One KUKA youBot picks up a baton placed in a predefined area of the room, and subsequently takes it to an area where it hands it over to a second youBot. This handover procedure takes place in motion while the 


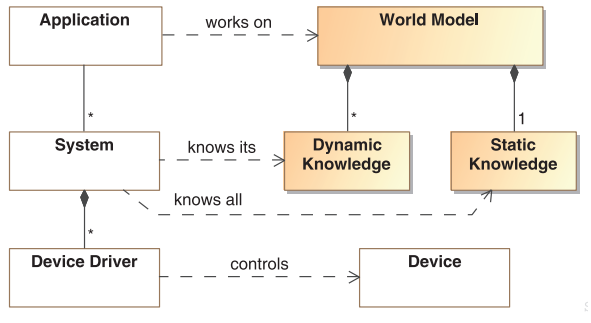

Figure 2. Software structure for distributed robots, adopted from [1].

youBots approach and pass each other. For this example, two youBots with laser scanners are used, along with a Vicon optical position tracking system. Both youBots are equipped with Vicon markers, while the baton is detected using the on-board laser scanners. Fig. 1 shows the two parts of the application, first picking up the baton and subsequently handing it over to the second youBot. The software for this example is implemented in an object-oriented fashion, representing the robots and baton as objects and correctly tracking their logical and geometric relationships during the process, so that the world model remains consistent with the real-world situation.

The remainder of the paper is structured as follows: In Sect. II, the overall approach is outlined. Then, the specification of logical relationships (Sect. III-A) and sensor meaning (Sect. III-B) is explained. Sect. IV details how the estimation is performed based on these specifications. In Sect. V, related approaches in theory as well as in existing robot frameworks are compared. To conclude the paper, Sect. VI explains the example implementation and experimental results and Sect. VII gives a conclusion and outlook.

\section{APPROACH}

When considering cooperating robots, the underlying software architecture influences how and where data is available. Fig. 2 (adopted from [1]) shows a way typical software architectures for distributed cooperating robots can be structured. Each robotic device is represented and controlled by a device driver which is defined as the component that communicates - usually in a real-time capable way - with the device through a vendor-specific interface. It has to forward control inputs to the device and receive feedback from the device. One or multiple device drivers belong to a system where all knowledge is shared between the components (no longer necessarily with real-time guarantees, e. g. ROS nodes belonging to the same master). Hence, all components within one system are allowed to access each other's provided data, as well as to communicate with and send commands to each other.

To perform a desired task, an application controls the involved systems to coordinate the work flow (e.g., two systems in the example of cooperating, but independent
youBots). Within an application, data is read from and commands are sent to controlled systems in order to have the corresponding devices execute the overall task. Each application performs its task based on its knowledge about controlled devices, systems and the environment. This includes geometric information such as positions and orientations of the relevant objects, as well as information about the structure of (parts of) the environment (e.g., topologies, maps), physical data (e.g., mass, friction) or shape data (e.g., 3D models). The world model data can be differentiated into dynamic and static knowledge. While static knowledge (e. g., given maps, shapes or physical data) is valid and available everywhere, dynamic knowledge (e. g., positions or sensor data) may be known in only one system or be different among different systems. The latter can be the case for the position estimation of mobile robots.

The environment of a robot consists of various physical objects, each at its respective position. The contribution of the presented approach is to propose how to model logical relationships between these objects, i. e., the topology of the known environment, as static knowledge in order to infer dynamic knowledge automatically and in a consistent way. Inferring a consistent world model is especially important in the case of multiple systems in one application, such as distributed robots that have to work together to achieve a task. For example, on-board sensors a robot is equipped with are only accessible in the system the robot is part of and, thus, can only be used for commands regarding this system. The responsibility for static knowledge, as it is the common part of the world model, lies at the application. Hence, the application manages topology changes, e. g., if and how a priori unknown objects are integrated into the topology.

Depending on the amount of structure in the environment, some geometric positions of objects are constant and can thus directly be modeled as static knowledge. Other positions however change over time and, thus, cannot be given exactly for an application that should be reusable later. Still, in order to work with them the application has to know about the existence of these objects and their logical relationships to further objects. Although these objects do have an exact position in the physical world, the application initially has no precise position information, which limits the amount of interaction that can be performed with these items.

To improve this situation, sensing can be employed to give the robot a glance of its environment. Based on sensor data, some positions (and velocities) of objects can be recovered, contributing to the geometry and, thus, dynamic knowledge of the application. To facilitate this, the interpretation of sensor measurements in a geometric context has to be defined. At application run-time, these interpretation definitions and incoming sensor data can be used to update the unknown or uncertain parts of the world model, so that it reflects a consistent interpretation of the received sensor data. In software, this process is performed through the introduction 


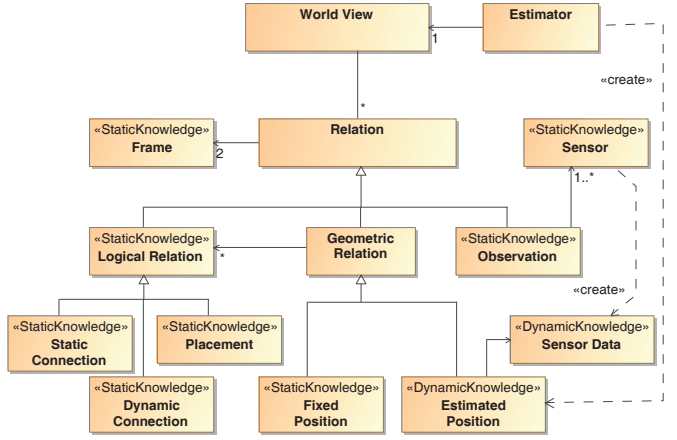

Figure 3. Concept model for describing both the static and dynamic knowledge of a robotic application using different kinds of Relations between spatial features of physical objects.

of logical and geometric relations, along with observations defining the semantics of sensor data and estimations derived from this information.

Fig. 3 gives an overview of the concepts used to express this information. A robot's or to be precise a system's knowledge about itself and its surrounding world is called a World View and consists of a set of Relations. A Relation correlates spatial features of objects like robots, workpieces or obstacles in the application. Such spatial features are modeled as Frames, which represent Cartesian coordinate systems. One type of Relations are Logical Relations. Those describe statically known logical correlations among objects' Frames. Objects that are connected to each other in a constant way, like a robot being mounted on a table, should be represented as Static Connections. Other tight, but variable connections, like the relation between a robot joint and its associated links, should be modeled as Dynamic Connections. For rather loose and changing correlations like objects being placed somewhere on the ground in the vicinity of another object, Placements are available.

The second type of Relations, Geometric Relations, form the geometric model and can describe the geometric position of an object relative to another, e.g., as a transformation matrix. Geometric Relations correspond to (sequences of) Logical Relations. They can either be constant (Fixed Position, e.g., for the position where the robot is mounted on the table), or be calculated from sensor values (Estimated Position).

However, Estimated Positions and their computation rule do not have to be defined explicitly, but may emerge automatically during application runtime: The application only has to define the interpretation of a sensor as Observations. Because an Observation is a special Relation, it describes a correlation between two Frames. Based on these $O b$ servations and the Logical Relations, so-called Estimators automatically derive ways to process sensors in order to recover geometric information about unknown positions, and provide them as computation rules for Estimated Positions

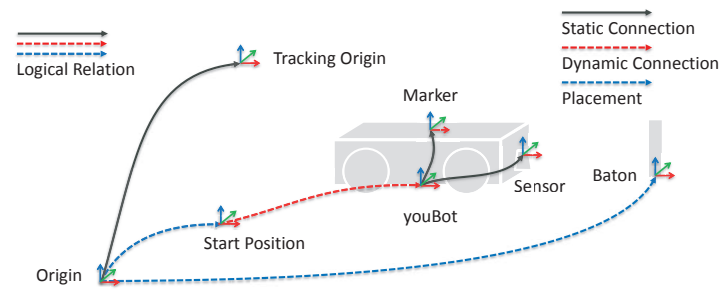

Figure 4. Logical model of the youBot environment

computed from Sensor Data.

\section{DEFINING THE LOGICAL MODEL AND SENSOR INTERPRETATION}

Basic ingredients of the world model are spatial features, called (coordinate) Frames. Each Frame represents a (named) position in space, including an orientation. However note that a Frame per se does not know its absolute position in space - the position is only defined relative to other Frames through Geometric Relations. Concerning these spatial features, a logical structure exists that is modeled in the logical model. Additionally, some features are part or target of sensor measurements which have to be interpreted in their corresponding context.

\section{A. Defining the Logical Model}

Between Frames, Relations can be established. As a basis of the environment model, Logical Relations describe that certain Frames are connected to each other, and include information about the durability of the link. The structure of Frames and Logical Relations forms an undirected graph, which allows navigation between different Frames if a sequence of Logical Relations exists that forms a path between the two Frames.

Fig. 4 gives an example of Frames and Logical Relations. Frames are depicted as named groups of arrows, which give the position as the intersection between the three arrows. Logical relations are shown as arrows between the Frames. The example shows Frames for a Start Position and a Tracking Origin which are linked to an Origin frame, along with a youBot that is linked to its Start Position. Additionally, a Marker frame representing the position that can be tracked by the optical tracking system is connected to the youBot, as well as the Sensor frame where the laserscanner is mounted. Additionally, a Baton is placed on the ground and thus connected to the Origin.

In this scenario, the different relationships have a different meaning, and are thus represented as different types of relationships. The position of the Tracking Origin relative to the Origin is fixed and given, and thus represented as a Static Connection. Similarly, the position of the Marker and Sensor relative to the youBot is fixed and constant. In contrast, the Start Position of the youBot in the world (relative to Origin) 


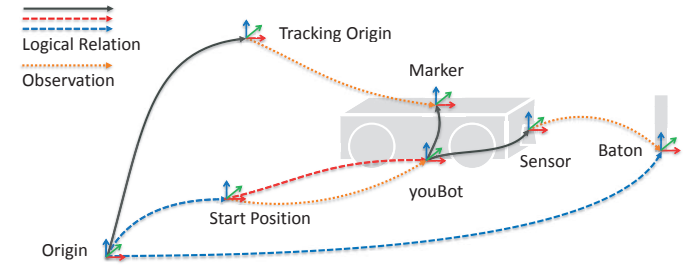

Figure 5. Excerpt of the World model for a youBot platform tracked using an optical tracking system.

is not fixed, but the youBot is rather placed into the world. Thus, this relationship is a Placement, as well as the one to the Baton. The position of the youBot relative to its Start Position is controlled by the youBot and is thus modeled as a Dynamic Connection. Consequently, Static Connection and Dynamic Connection represent persistent relations that will exist for the entire run time of an application, while Placements are transient and can be removed. Placements and Static Connections are assumed to model a relationship with constant transformation, while the transformation of Dynamic Connections varies over time.

When the baton is grasped by the youBot, the Placement from Origin to Baton is removed, and a new Placement from the Gripper to the Baton is established. This topology change that occurs at application run time has an influence on the geometric relations and is detailed in the following sections.

\section{B. Defining the Sensor Interpretation}

To derive a geometric model elaborating the logical model defined above, Geometric Relations providing transformations and velocities have to be added. For relations that are not given statically, this transformation often has to be derived from sensor measurements. To achieve this, the semantics of measured sensor data have to be defined in the form of Observations that describe that a certain aspect of the World model is measured by a given sensor, or can be calculated from given Sensor Data. For the scope of this paper, we limit ourselves to measurements that provide the full transformation and velocity between two Frames, however not limited to Frames that are directly connected by logical relations. For more complex cases, measurements could e.g. also define partial information such as the distance between two Frames or the distance to a given plane (e.g. for the height of a quadcopter) or give information about velocities and accelerations only.

Looking into robot environments, different types of sensors and correspondingly observations occur: For logical relations inside Actuators, most can be measured through proprioceptive sensors. Then, the sensor semantics can be defined following the logical structure of the object: In the case of the youBot base, observations can be defined

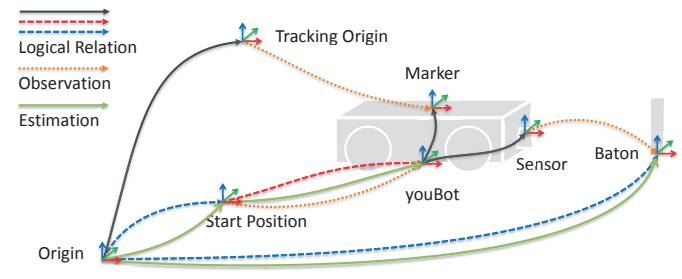

Figure 6. Estimations established for a youBot platform when all sensors are available

that describe the position of the wheels relative to their wheel mount frames using sensor data provided by the wheel encoders. Similarly, the transformation between Start Position and the youBot is provided through odometry calculations based on wheel encoders, so the observations describe transformations that follow a logical relation.

For other logical relations, however, this is not the case: Assuming the youBot platform is driving on the floor starting at a position not exactly known, application geometry is usually modeled as a Placement from the Origin frame to the youBot Start Position (cf. Fig. 4). Its transformation however cannot be measured directly, because the Start Position is an intermediate concept that does not have a direct representation in the physical world (at least after the youBot platform has moved). Fig. 5 as an extension to Fig. 4 gives an overview of this situation. It shows a youBot platform on the floor that is tracked through an optical tracking system. In addition to the logical model, three observations are given (represented as dotted arrows). As mentioned above, the position of the youBot relative to its Start Position is given by odometry sensors. Additionally, the Marker of the youBot is tracked using the tracking system, so the observation between Tracking Origin and Marker is given by the tracking system. Furthermore, the Baton is tracked from the Sensor through the laserscanner (with some post-processing). The transformations defined by the second and third observation cannot directly be used to describe the transformation of a Logical Relation, but first have to be converted.

Looking at the three observations given here, the first and third are only applicable on the youBot system, because they use sensor data that is only available to the youBot. The second observation however can be used wherever the tracking system's data is available, e.g. for a second cooperating youBot.

\section{DERIVING GEOMETRIC INFORMATION THROUGH ESTIMATION}

To coordinate the interplay between logical relations and observations and to provide estimations for the uncertainties present, the concept of Estimators on the system level is introduced. An Estimator listens to changes in the logical 


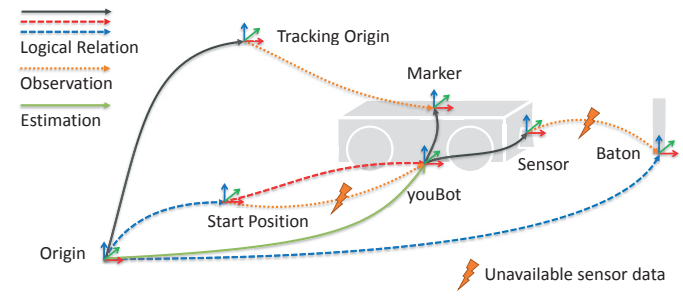

Figure 7. Estimations established for a youBot platform from an outside view if only tracking data is available

relations as well as observations, and augments its World View with Estimated Positions that reflect one Logical Relation or shortcut multiple ones. These Estimated Positions can be seen as estimation pipelines processing the data provided by sensors as defined in the observations, giving computation rules for how to calculate the transformation and velocity if the sensor's dynamic knowledge is available in the corresponding system.

Given the example in Fig. 6, the Estimator creates three Estimated Positions: The first relation goes from the Start Position to the youBot frame and takes the value from the odometry sensor as a transformation. The second relation from Origin to Start Position is a bit more complex: The transformation can be combined from the transformation from Origin to Tracking Origin, followed by the transformation provided by the Observation, the transformation from the Marker to the youBot and the transformation from youBot to Start Position. For the last part, the transformation provided by the first estimated Relation has to be used. Similarly, the third transformation can be computed using the observed position of the Baton, along with the estimated position of the youBot. However, when another robot observes the youBot from outside, the sensor data for the odometry Observation as well as the laserscanner measurements are not available. In this case, the Estimator has to create an Estimated Position directly from the Origin frame to youBot (cf. Fig. 7).

\section{A. Establishing and Updating the Geometric Model}

At application run-time, Estimator implementations work as listeners that react to changes in the Observations, Logical and Geometric Relations. The Estimator tries to find cycles in the graph formed by Logical Relations and Observations, and uses their information to build new known Estimated Positions. In cycle search, it tries to minimize the number of Logical Relations without corresponding Geometric Relations required to form estimations in order to keep the estimations structurally as close to the logical structure as possible. The resulting cycle then consists of an Observation, a (maybe empty) sequence of Geometric Relations, followed by a sequence of Logical Relations (that will be estimated and should thus be as short as possible), and a (possibly

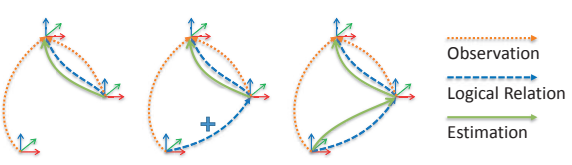

(a) Adding a Logical Relation

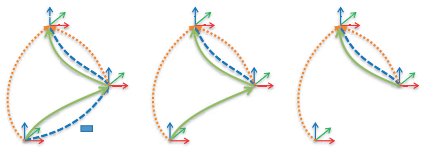

(b) Removing a Logical Relation

Figure 8. Estimation modifications when changing Logical Relations

empty) sequence of Geometric Relations closing the loop. For a found cycle, the Estimator takes the Observation's transformation and velocity and converts it for the Frames forming the start and end of the Logical Relation sequence, so that calculation rules describing the overall behavior (position and velocity) of the Logical Relation sequence are available. These calculations are then used to define the Estimated Position, and subsequently evaluated whenever this aspect of the World model is accessed by the application, e.g. during motion planning.

When a Logical Relation is added (cf. Fig. 8(a)), an Estimator checks if any of its known Observations can be used to form a cycle in the World view including the new Logical Relation. If so, an Estimated Position is established based on the Logical Relation and Observation. For Geometric Relations added, the Estimator checks if the new Geometric Relation has an effect on any of the existing estimations. This is the case when the Relation influences the first or last Logical Relation resolved through the estimation, causing the Estimated Position to be recreated based on the new situation. When a Logical Relation is removed (cf. Fig. 8(b)), the corresponding estimations become invalid and are removed. Similarly, removing Geometric Relations can invalidate estimations if they occurred in the cycle used to build the estimation, so new estimations for the corresponding logical connections have to be built.

Adding an Observation (cf. Fig. 9(a)) may allow resolving

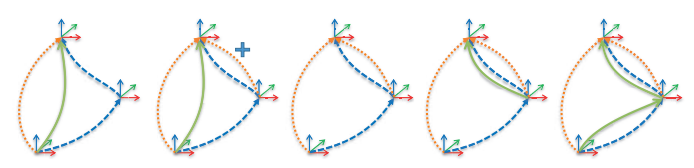

(a) Adding an Observation

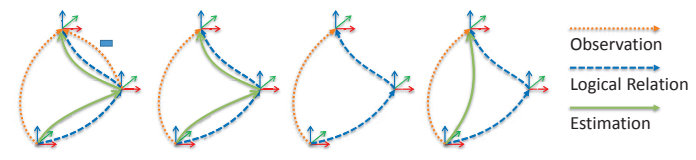

(b) Removing an Observation

Figure 9. Estimation modifications when changing Observations 
one of the Logical Relations by forming a cycle including the new Observation and building a corresponding estimation. If the resulting estimation contains a subset of the Logical Relations used in another estimation, the latter estimation is removed and recreated with the option to use the newly built estimation, bringing the estimations closer to the logical structure (e.g. in the last step of Fig. 9(a)). When an Observation is removed that has been used by estimations (cf. Fig. 9(b)), the corresponding Estimations are removed and new cycles for the corresponding Logical Relations are searched.

As an example, a workpiece tracked by a sensor is considered: The workpiece lying on the floor is connected to the ground using a Placement. Additionally, an Observation is given that provides the position of the workpiece relative to the sensor, and thus allows the calculation of a transformation for the Placement. However, once the workpiece is grasped, the Placement on the ground is removed and another Placement to the gripper is added. In this situation, the same Observation now has to be used to provide another Estimated Position. This case is automatically handled by Estimators.

\section{B. Handling Delayed and Infrequent Data}

In simple cases, the Estimator can assume that all sensors used in Observations are precise and provide values all the time, without any time delay. Then, the estimator can use the latest position data from all Observations to define its Estimated Positions.

For Observations referencing Sensor Data that are only provided sporadically or delayed, a more complex Estimator is required, however still following the method outlined in Sect. IV-A. It still assumes that all Observations are precise, but accepts that some Observations are only provided infrequently, but with correct time stamps (as seen by the tracking system when an object is temporarily lost or a lag in wireless network communication occurs). It further respects the types of Logical Relations. For Placements and Static Connections, it assumes that they are actually constant and only have to be changed to correct measurement errors (which is true for objects placed on the ground, as well as for the Start Position of a youBot), while for Dynamic Connections extrapolating with constant velocity is an appropriate estimation. In this case, Estimated Positions are created between the same Frames as in the simple case, however, transformations are taken from a consistent time snapshot and extrapolated if required. While supporting more use cases, this estimator has the disadvantage of increased memory usage and computation time: To calculate estimations for a consistent time, it has to keep track of previous values and times of the sensor data, and has to select a corresponding time to use the data from. Especially on resource-constrained systems that need an estimator that runs at a high frequency with hard real-time guarantees, using a simple estimator instead can thus be a better option when no greater time delays are to be expected for the sensor data.

\section{RELATED WORK}

Looking at control theory, the process of integrating sensor data into the world model is similar to the concept of state observers used along with the state-space representation of a system model: A system model consists of a state equation describing the behavior of the system under the influence of the given system inputs, and an output equation that defines the relationship between system state and the measured variables. Standard estimation methods such as the Kalman Filter [2] for linear problems allow processing system inputs and measurements to recover the state of the system, tracking the uncertainty of the present state variables. For non-linear problems (that occur in robotics, e.g. through rotations that have an non-linear effect on the robot position when the robot drives forward), extensions of the Kalman Filter [3] are available such as the Extended Kalman Filter linearizing the problem around the given state, or the Unscented Kalman Filter that samples chosen points in the probability distribution. Furthermore, Particle Filters [4] allow tackling problems with greater uncertainty, such as an initial localization of a robot in a known map. These methods can also form the basis of further Estimators for use in the proposed approach, introducing the handling of uncertainty and Gaussian noise.

Looking at $R O S$, the frame graph is typically managed through the if library [5], where a tree of geometric relationships between frames can be established. However, no semantic information is contained there, apart from a separation between static and dynamic transformations (through the $/ t f \_s t a t i c$ and $/ t f$ topics). Estimation and sensor integration for robot positions is typically performed through specialized components. According to ROS Enhancement Proposal 105 [6], mobile platforms should be modeled with three distinguished frames: The frame map represents the origin of the robot environment (and a corresponding map), while odom represents the starting point of the robot and base denotes the robot itself. The transformation between map and base does not have to be continuous, but may not drift over time, while the transformation between odom and base has to be continuous, but may exhibit unbounded drift. In the frame tree used in ROS, odom is the parent frame of base, while map is the parent of odom. This modeling is similar to the model used in the proposed approach, with odom representing the Start Position and map representing a fixed frame (such as the Tracking Origin or Origin). To handle sensor data, robot_pose_ekf [7] and later robot_localization as described by Moore et al. [8] provide different estimation algorithms as ROS Nodes, including an Extended and Unscented Kalman Filter. These Nodes can process various sensor data, including global positioning 


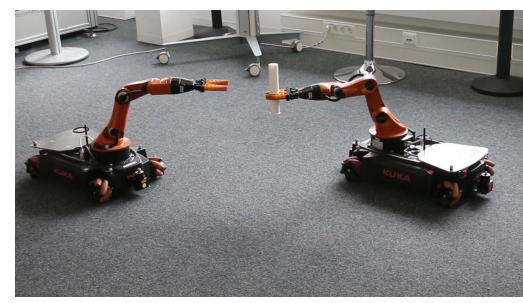

(a) Situation before handover

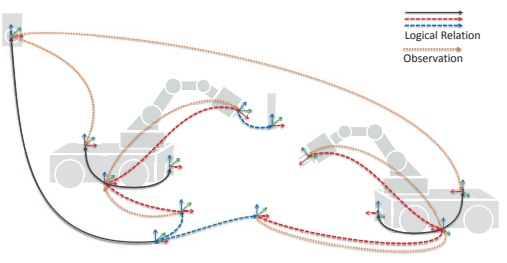

(d) Model before handover

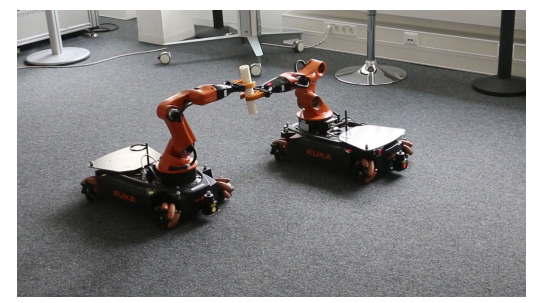

(b) Situation during handover

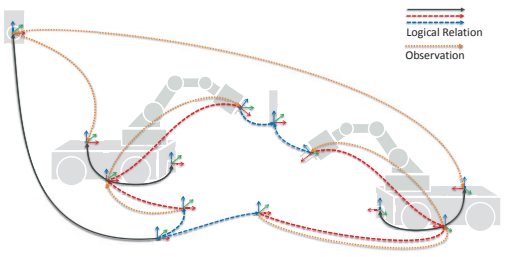

(e) Model during handover

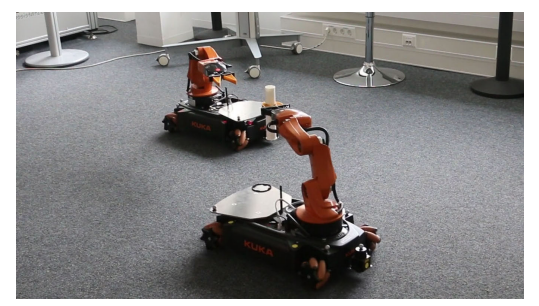

(c) Situation after handover

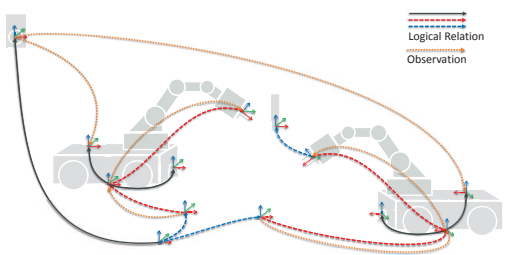

(f) Model after handover

Figure 10. Passing the baton - model and reality

systems (GPS), inertial measurement units (IMU) and odometry (ODOM) data, and publish the transformation between map and odom or the transformation between odom and base. However, these estimation nodes have to be configured manually, and cannot be used with topology changes: For an object first tracked using multiple sensors, and then grasped by a robot, the parent frame changes, and thus the estimation node has to be reconfigured.

In OROCOS, sensor data and estimation can e.g. be handled through the $i T a S C$ framework [9]. There, uncertainty can be defined for object or feature coordinates, which is then processed during task execution using standard estimation techniques (as introduced above). However, this world model and observation uncertainty model is tied to one given task and is only in effect during task execution. In contrast, in the proposed framework, the logical model, uncertainties and observations can be defined globally and independently, which allows sensor data processing between the execution of different tasks and the derivation of uncertainty and measurement models for a given task from the global model.

\section{EXPERIMENTAL RESULTS}

The concepts introduced in this paper have been evaluated in multiple scenarios, one of which uses two cooperating youBots. On the hardware side, each youBot is equipped with a bionic soft gripper [10] mounted at the arm, a WiFi adapter, as well as with retro-reflective markers to provide position tracking. On the system level, both youBots are controlled as separate systems without implicit data transfer, based on a C++ implementation of the Robot Control Core [11] running the onboard computer under Linux with Xenomai extensions to achieve real-time control. However, they are allowed to receive Vicon tracking data through WiFi (that sometimes exhibits time delays), as well as tasks from an application. On the application level, a single application is used, written in Java based on the Robotics API [12], executed on a standalone computer. However, this application could as well have been executed on any of the youBots.

In this application, a common world model is defined, consisting of the logical model as well as the available sensors and their observations as detailed in the previous sections. For picking up the baton, the raw distance readings of the laser scanner are first filtered, limiting them to distance readings that are within a radius of $50 \mathrm{~cm}$ around the expected pick up position. Then a pole detection algorithm is used that searches for clusters of a length corresponding to the expected diameter of the baton. The detected cluster position is used as Sensor Data for the Observation of the Baton. Based on the (dynamically estimated) position of the baton, the pickup process is executed. For passing the baton, the application commands the youBot platforms to pass each other, while the gripper is commanded to move towards the center position between the two youBots. Once the distance between the youBots is sufficiently small, the receiving youBot closes its gripper to grasp, followed by the other youBot releasing the baton. When both youBots reach a certain distance after passing each other, the arm motion is stopped, completing the task. These tasks are specified on the full world model, while for execution for each system the tasks are translated into a representation that only uses available data. This way, for each system a corresponding view is automatically derived at run-time and used for command execution, using only the sensor data and estimations available at each youBot.

Fig. 10 shows pictures of an example run ${ }^{1}$ of the handover procedure, along with the logical relations and observations present at the corresponding times. During execution, the

\footnotetext{
${ }^{1} \mathrm{~A}$ video is available at http://video.isse.de/consistentworldmodel
} 
available estimated positions differ between both youBots: While each youBot knows its start position, it does not know the start position of the other youBot (cf. Fig. 6 and 7). Of course, both youBots could have resorted to a model where the Vicon sensor measurement is used to calculate the position of the youBot relative to the Origin (cf. Fig. 7). However, using a more fine-grained model where available (Fig. 6) provides better performance when the tracking system fails: Whereas in this case no further information about motion is available through the Vicon system, the motion of the youBot relative to its start position can still be tracked using odometry, assuming that the start position remains stationary relative to the Origin. This estimation is better than a simple extrapolation of the previous motion based on a constant velocity or acceleration scheme (which has to be applied in the case of Fig. 7), because it takes into account the measured wheel revolutions, and can thus handle non-uniform motions.

To show the utility of this approach and the possible reuse, the same application was used in another system setup: There, both youBots were configured to be in a single (simulation) system. In this case and without modifying the application or any estimation configuration, at run time only a single world view was created for the simulation system, including estimation models for both youBots similar to Fig. 6. Also in this scenario, the baton could be handed over. Further application examples using the proposed concepts are explained in [13].

\section{CONCLUSION}

In this paper, we introduced a separation between logical, geometric and measurement information for robotic world models. Logical relationships describe correlations of physical objects via their spatial features which can be reused in different use cases or even applications. Moreover, such correlations are often an inherent part of the model of a robotic device that thus only have to be modeled once. Using the introduced concepts of Observations as measurement definition based on spatial features and Estimators to dynamically integrate the sensor data as geometric information at run-time can be seen as a powerful modeling tool for robot programming, allowing the specification of relationships that can be used by different estimation techniques. An important contribution of this paper is that this separation automatically helps to create consistent world views when dealing with distributed robot systems or changing environments, because changes to the logical model are reflected in all World views.

Apart from continuous computations at run-time, the modeled relationships can additionally be used for offlineprocessing, allowing parameter estimation based on recorded sensor values through non-linear optimization. Possible use cases here are to determine the exact position of a tracking marker relative to the robot it is attached to, or the calibrate a robot by performing certain motions, recording the sensor data and optimizing the system parameters in a way to minimize the difference between observations and system model.

\section{REFERENCES}

[1] A. Schierl, A. Angerer, A. Hoffmann, M. Vistein, and W. Reif, "On structure and distribution of software for mobile manipulators," in Informatics in Control, Automation and Robotics; 12th International Conference, ICINCO 2015; Colmar, France, July 21-23, 2015; Revised Selected Papers, ser. LNEE, J. Filipe, K. Madani, O. Gusikhin, and J. Sasiadek, Eds. Springer, 2016, vol. 383, pp. 209-228.

[2] R. E. Kalman, "A new approach to linear filtering and prediction problems," Journal of Fluids Engineering, vol. 82, no. 1 , pp. 35-45, 1960.

[3] S. Julier, J. Uhlmann, and H. Durrant-Whyte, "A new approach for filtering nonlinear systems," in Proceedings of the 1995 American Control Conference, vol. 3, Jun 1995, pp. $1628-1632$.

[4] B. Arulampalam, Beyond the Kalman Filter: Particle Filters for Tracking Applications. Artech House, 2004.

[5] T. Foote, "tf: The transform library," in Proceedings of the 2013 IEEE International Conference on Technologies for Practical Robot Applications (TePRA 2013), Apr 2013, pp. 1-6.

[6] W. Meeussen. (2010, Oct) Coordinate frames for mobile platforms. Online, accessed Feb 2016. [Online]. Available: http://www.ros.org/reps/rep-0105.html

[7] W. Meeussen and D. V. Lu. robot_pose_ekf. Online, accessed Feb 2016. [Online]. Available: http://wiki.ros.org/robot_pose_ekf

[8] T. Moore and D. Stouch, "A generalized extended kalman filter implementation for the robot operating system," in Proceedings of the 13th International Conference on Intelligent Autonomous Systems (IAS-13). Springer, Jul 2014.

[9] J. De Schutter, T. De Laet, J. Rutgeerts, W. Decré, R. Smits, E. Aertbeliën, K. Claes, and H. Bruyninckx, "Constraintbased task specification and estimation for sensor-based robot systems in the presence of geometric uncertainty," The International Journal of Robotics Research, vol. 26, no. 5, pp. 433-455, 2007.

[10] BionicTripod with FinGripper - versatile movement and adaptive grasping. Online, accessed Feb 2016. [Online]. Available: https://www.festo.com/cms/de_corp/9779.htm

[11] M. Vistein, A. Angerer, A. Hoffmann, A. Schierl, and W. Reif, "Flexible and continuous execution of real-time critical robotic tasks," International Journal of Mechatronics and Automation, vol. 4, no. 1, pp. 27-38, Jan 2014.

[12] A. Angerer, A. Hoffmann, A. Schierl, M. Vistein, and W. Reif, "Robotics API: Object-Oriented Software Development for Industrial Robots," Journal of Software Engineering for Robotics, vol. 4, no. 1, pp. 1-22, 2013.

[13] A. Schierl, "Object-oriented modeling and coordination of mobile robots," PhD thesis, Universität Augsburg, 2017. 